\title{
Impacto das Variações no Crédito Rural e no Investimento em Pesquisa Agrícola na Produtividade da Agricultura Brasileira Contemporânea ${ }^{1}$
}

\author{
Celso José Costa Junior ${ }^{2}$
}

\begin{abstract}
Resumo: A queda da produtividade das principais lavouras pode estar relacionada à redução dos investimentos em pesquisa. Ainda, o crédito rural subsidiado pode ser necessário para um aumento da produtividade, uma vez que esse é um fator essencial para a produção e para a modernização setorial. Percebendo a relevância desses dois pontos, este trabalho utiliza um modelo DSGE com economia fechada, sem governo e com dois setores. Os resultados apontam que o baixo investimento em pesquisa levou à baixa produtividade do setor agrícola até 2011, que se recuperou em 2012 e 2013. Por outro lado, o crédito rural subsidiado apresentou resultado nulo até 2009 e, em 2010 e 2011, o resultado foi positivo, mas tornou-se negativo em 2012 e 2013. Entre as duas políticas propostas, o subsídio ao crédito rural apresentou melhor resultado do que a pesquisa agrícola, pois o desempenho do produto foi superior no primeiro caso.
\end{abstract}

Palavras-chaves: Modelo DSGE, crédito rural subsidiado, pesquisa agrícola.

\begin{abstract}
The fall in productivity of the main crops may be related to the reduction of investments in research. Moreover, subsidized rural credit may be necessary for an increase in productivity, since this is a key factor for production and sectoral modernization. Realizing the relevance of these two points, this work uses a DSGE model with closed-economy, no government, and two-sector. The results indicate that low investment in research led to low productivity of the agricultural sector until 2011, which recovered in 2012 and 2013. On the other hand, subsidized rural credit presented zero results until 2009, and in 2010 and 2011, the result was positive, but became negative in 2012 and 2013. Among the two policies proposed, the rural credit subsidy presented better results than agricultural research, because the performance of the product was higher in the first case.
\end{abstract}

Key-words: DSGE model, subsidized rural credit, agricultural research.

Classificação JEL: C11, C60, Q10.

DOI: http://dx.doi.org/10.1590/1234-56781806-94790560401

1. Data de submissão: 15 de junho de 2016. Data de aceite: 23 de fevereiro de 2018.

2. Universidade Estadual de Ponta Grossa (UEPG), Ponta Grossa (PR), Brasil. E-mail: cjcostaj@yahoo.com.br 


\section{Introdução}

Certamente, a produtividade está entre os pontos mais relevantes para a produção agrícola, pois está relacionada à produção mais eficiente e a menores custos dos alimentos e dos produtos agrícolas em geral. Contudo, sua mensuração é sensível ao método escolhido, à sua definição, às distintas bases de dados disponíveis, às culturas e áreas agrícolas e a fatores sistêmicos, tais como: custos dos insumos, infraestrutura de transporte, canais e arranjos de distribuição, inclusive de venda para o exterior (FREITAS, 2014).

A queda da produtividade das principais lavouras pode estar relacionada à redução dos investimentos em pesquisa, o que resultaria em menor quantidade de inovações no setor. Ainda, o crédito rural subsidiado pode ser necessário para um aumento da produtividade, uma vez que esse é um fator essencial para a produção e para a modernização setorial. Como exemplo, a transformação de insumos em produtos ocorre com certa defasagem de tempo, a disponibilidade de crédito fornece liquidez e possibilita aos seus usuários a aquisição de insumos de melhor qualidade, acelera a adoção de melhores tecnologias e possibilita a ampliação da escala de produção pela aquisição de mais terras ou novos equipamentos (GASQUES et al., 2012a).

O objetivo deste trabalho é contribuir com uma análise dos fatores que estariam relacionados ao aumento da produtividade e ao desempenho da agricultura destacados no parágrafo anterior. Para tanto, será usado um modelo DSGE com economia fechada, sem governo e com dois setores, sendo que o setor agrícola incorpora a utilização de crédito rural subsidiado e a pesquisa agrícola. As contribuições específicas esperadas com este trabalho são: disponibilizar um modelo apoiado na macroeconomia moderna para discussão de assuntos particulares da literatura econômica agrí- cola e, assim, ajudar futuras pesquisas com interesse nessa abordagem empírica; e apresentar uma função de acúmulo de conhecimento agrícola, ferramenta que tem origem na literatura de crescimento endógeno, mas não utilizada na modelagem DSGE.

A produtividade agrícola apresentou baixo desempenho de 1947 a 1965 devido ao baixo nível de utilização de fertilizantes (HERMANN, 1972). No período seguinte, com duração até 1995, um terço do crescimento da produção agrícola esteve relacionado à produtividade (BARROS, 1999). Segundo Brigatte e Teixeira (2012), a performance dessa variável relaciona-se com a pesquisa agrícola daquela época. De 1995 a 2006, a produtividade foi ainda mais significativa, segundo Gasques et al. (2012b), 68\% do crescimento do produto agrícola foi resultado do aumento da produtividade e $32 \%$, de aumentos nos insumos.

Em Gasques et al. (2012a), entre 1975 e 2011, houve um aumento do índice de utilização de terras, redução do índice de pessoal ocupado e elevação do capital (máquinas, defensivos e fertilizantes). Os resultados de elevação da produção agrícola total, com menor emprego de mão de obra, ancoram-se na incorporação de capital em termos de máquinas e em alterações nos parâmetros tecnológicos das funções de produção em uso nas atividades agrícolas empreendidas no País (FERREIRA et al., 2006; BONELLI e FONTES, 2013).

$\mathrm{O}$ modelo usado neste trabalho é um novo-keynesiano com economia fechada, sem governo e com dois setores: agrícola e não agrícola. Outras duas características são particulares deste modelo: crédito rural usado para o pagamento de capital de giro ${ }^{3}$ e a pesquisa agrícola ${ }^{4}$. O subsídio ao crédito rural e o investimento em pesquisa são financiados por um "tri-

3. Como em Cooley e Quadrini (1999), Fuerst (1992) e Carlstrom e Fuerst (1995).

4. Ideia apoiada em Romer (1990). 
buto" lump-sum junto às famílias. Assim, a finalidade geral desse modelo é testar se essas duas características influenciaram a produtividade e o desempenho da agricultura brasileira contemporânea.

Além dessa introdução, o modelo está estruturado com apresentação do modelo na seção dois, a terceira seção relata os procedimentos de tratamento de dados e estimação dos modelos, na quarta seção são apresentados os resultados, e então o trabalho é finalizado com a conclusão dos resultados.

\section{Modelo}

Este trabalho é apoiado em um modelo novo-keynesiano padrão com economia fechada, sem governo e com dois setores: agrícola e não agrícola. Entre as características particulares, o modelo possui crédito rural subsidiado e investimento em pesquisa agrícola. A estrutura geral do modelo é apresentado na Figura 1.

Intratemporalmente, as famílias adquirem bens de consumo agrícola e não agrícola e decidem a quantidade de horas que irão trabalhar. Ainda precisam decidir entre consumo presente e consumo futuro; para tanto, podem poupar adquirindo bens de capital (investimento) ou títulos financeiros (empréstimos para capital de giro das firmas).

As firmas utilizam crédito para financiar capital de giro para pagamento de trabalho; entretanto, as firmas produtoras de bens agrícolas podem financiar parte desse serviço utilizando crédito rural subsidiado. Já o investimento em pesquisa agrícola é usado para melhorar a produtividade aumentadora do trabalho no setor agrícola. Essas duas políticas são financiadas por um "tributo" lump-sum sobre as famílias.

Figura 1. Estrutura do modelo

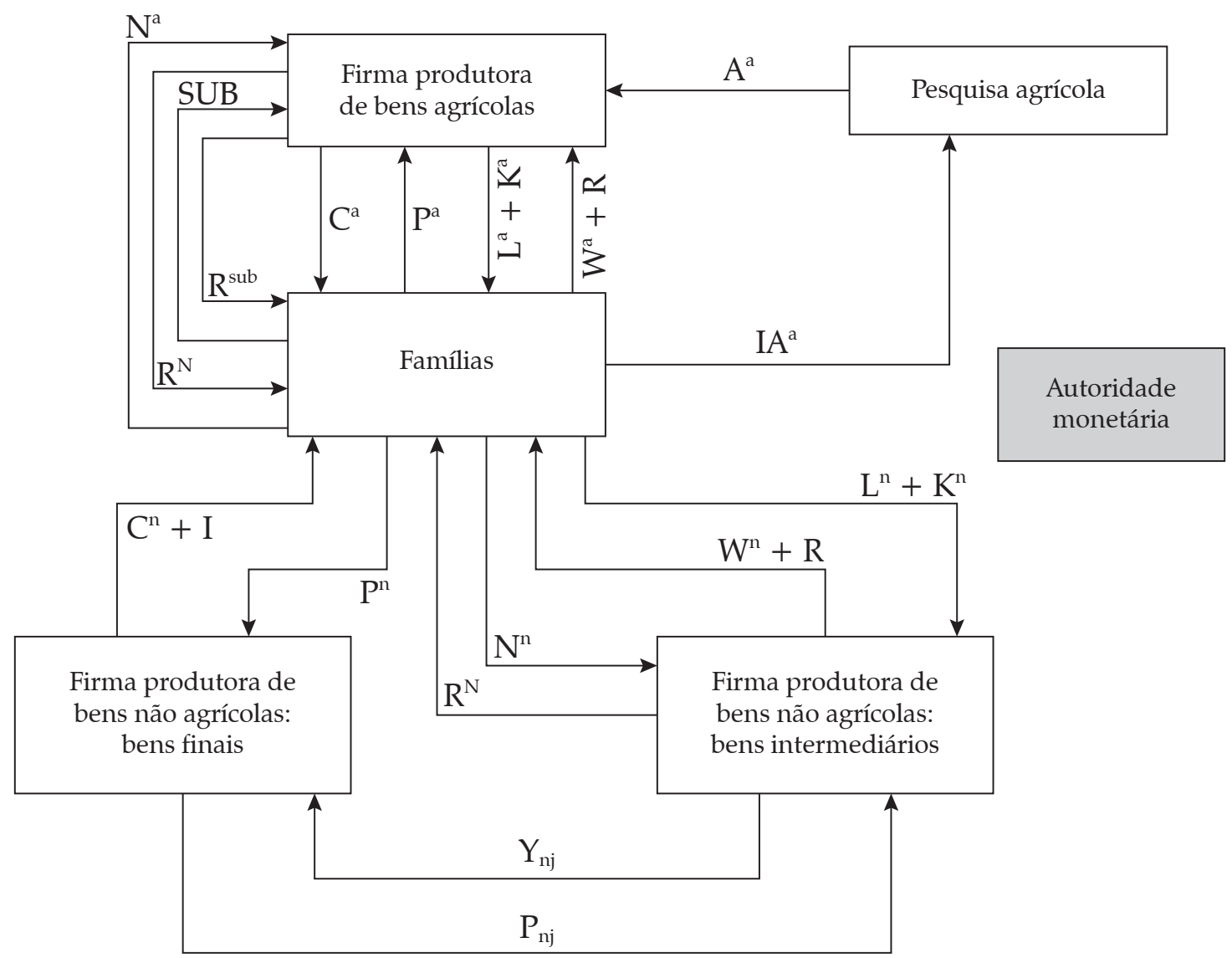

Fonte: Elaboração própria. 
554 - Impacto das Variações no Crédito Rural e no Investimento em Pesquisa Agrícola na Produtividade da Agricultura Brasileira Contemporânea

\subsection{Famílias}

Há um continuum de famílias indexadas por $j \in[0,1]$. Essa família representativa maximiza a sua função utilidade intertemporal escolhendo consumo, poupança e lazer:

$$
\max _{C_{t}^{a}, C_{t}^{n}, L_{t}, N_{t+1}, K_{t+1}} E_{t} \sum_{t=0}^{\infty} \beta^{t} S_{t}^{P}\left(\frac{C_{t}^{1-\sigma}}{1-\sigma}-S_{t}^{L} \frac{L_{t}^{1+\varphi}}{1+\varphi}\right)
$$

sujeita à seguinte restrição orçamentária

$$
\begin{aligned}
& C_{t}^{a} P_{t}^{a}+\left(C_{t}^{n}+I_{t}\right) P_{t}^{n}+N_{t+1}=W_{t} L_{t}+R_{t} K_{t}+ \\
& +R_{t-1}^{N} N_{t}-\left(S U B_{t+1}-R^{S U B} S U B_{t}\right)-I A_{t}^{a}
\end{aligned}
$$

Em que $E_{\mathrm{t}}$ é o operador de expectativas, $\beta$ é o fator de desconto intertemporal, $\sigma$ é o parâmetro de aversão ao risco relativo, $\varphi$ é a desutilidade marginal do trabalho, $C$ é o consumo agregado, $C^{a}$ é o consumo de bens agrícolas, $C^{n}$ é o consumo de bens não agrícolas, $I$ é a aquisição de bens de investimento, $P^{a}$ é o preço dos bens agrícolas, $P^{n}$ é o preço dos bens não agrícolas, $N$ é a poupança das famílias em ativos financeiros, $W$ é o salário, $R$ é o retorno do capital, $R^{N}$ é a remuneração

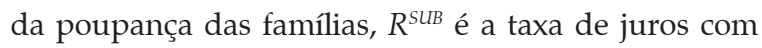
subsídio, SUB e IAa representam os "tributos" lump-sum para subsídio ao crédito rural e para pesquisa agrícola, respectivamente. Em outras palavras, no período $t$, a família deve direcionar parte dos seus recursos para crédito rural ${ }^{5}$ e para investimento em pesquisa agrícola (não recebendo nenhum benefício direto por isso).

Os bens de consumo agrícola e não agrícola são agregados pela seguinte função CES:

$$
C_{t}=\left[\omega a\left(C_{t}^{a}\right) \frac{1}{\psi a}+(1-\omega a)\left(C_{t}^{n}\right) \frac{1}{\psi a}\right]^{\psi a}
$$

Em que $\omega a$ é a participação dos bens de consumo agrícola em relação ao consumo agregado e $\psi a$ é a elasticidade de substituição entre esses dois tipos de bens de consumo.

O capital segue a seguinte regra de movimento:

$$
K_{t+1}=(1-\delta) K_{t}+I_{t}
$$

Em que $\delta$ é a taxa de depreciação do capital.

5. Sendo que esses recursos são remunerados por uma taxa de juros menor do que a taxa de juros de mercado $\left(R^{S U B}<R^{N}\right)$.
Também existem dois choques relacionados à demanda agregada do modelo. $S^{P}$ é o choque de preferência intertemporal usado para capturar alterações nos interesses de consumo das famílias no curto prazo. E $S^{L}$ é o choque de oferta agregada usado para representar alterações na predileção ao lazer desse agente.

$$
\begin{aligned}
& \log S_{t}^{P}=\rho_{P} \log S_{t-1}^{P}+\varepsilon_{P, t} \\
& \log S_{t}^{L}=\rho_{L} \log S_{t-1}^{L}+\varepsilon_{L, t}
\end{aligned}
$$

Em que $\rho_{P}$ e $\rho_{L}$ são os componentes autorregressivos desses dois choques, enquanto $\varepsilon_{p, t} \sim N\left(0, \sigma_{p}\right)$ e $\varepsilon_{L, t} \sim$ $N\left(0, \sigma_{L}\right)$.

O modelo não possui uma instituição financeira formal, de tal modo que o total de crédito da economia é fornecido pelas famílias. Espontaneamente, a família escolhe a quantidade de crédito que irá fornecer as firmas (agrícolas e não agrícolas) dada uma taxa de juros de mercado $R^{N}$.

$$
N_{t+1}=\left(1-\theta^{\text {SUB }}\right) L_{t}^{a} W_{t}^{a}+L_{t}^{n} W_{t}^{n}
$$

Por outro lado, compulsoriamente, as famílias fornecem crédito subsidiado ao setor agrícola:

$$
S U B_{t+1}=\theta^{\text {SUB }} L_{t}^{a} W_{t}^{a} S_{t}^{\text {SUB }}
$$

Esse crédito subsidiado possui um componente estocástico dado pela seguinte função AR:

$$
\log S_{t}^{\text {SUB }}=\rho_{\text {sUв }} \log S_{t-1}^{S U B}+\varepsilon s \backsim B, t
$$

Em que $\theta^{\text {SUB }}$ é a participação do crédito subsidiado em toda necessidade de capital de giro da firma produtora de bens agrícolas e $\varepsilon_{S U B, t} \sim N\left(0, \sigma_{S U B}\right)$.

As condições de primeira ordem para o problema da família são:

$$
\begin{aligned}
& C_{t}^{a}=C_{t}^{n}\left(\frac{P_{t}^{n}}{P_{t}^{a}}\right)^{\frac{1}{1-\psi a}} \\
& S_{t}^{P} L_{t}^{\varphi} C_{t}^{-\chi}\left(C_{t}^{a}\right)^{1-\psi a}=\frac{W_{t}}{P_{t}^{a}}
\end{aligned}
$$

Em que $\chi=\left(\frac{1-\psi a}{\psi a}\right)-\sigma$.

$$
\left(\frac{S_{t}^{P} C_{t}^{\chi}\left(C_{t}^{a}\right)^{\psi a-1}}{R_{t}^{N} P_{t}^{a}}\right)=\beta E_{t}\left(\frac{S_{t+1}^{P} C_{t+1}^{\chi}\left(C_{t+1}^{a}\right)^{\psi a-1}}{P_{t+1}^{a}}\right)
$$




$$
\begin{aligned}
& \left(\frac{S_{t}^{P} C_{t}^{\chi}\left(C_{t}^{a}\right)^{\psi a-1}}{P_{t}^{a}}\right)= \\
& =\beta E_{t}\left\{\left(\frac{S_{t+1}^{P} C_{t+1}^{\chi}\left(C_{t+1}^{a}\right)^{\psi a-1}}{P_{t+1}^{a}}\right)\left[(1-\delta) P_{t+1}^{n}+R_{t+1}\right]\right\}
\end{aligned}
$$

A equação (10) representa o consumo relativo entre bens agrícolas e não agrícolas, a equação (11) é a oferta de trabalho, e as equações (12) e (13) são funções de Euler para a poupança em ativos financeiros e em ativos de capital, respectivamente.

\subsection{Firmas}

As firmas estão divididas em dois setores: agrícola e não agrícola.

\subsubsection{Firmas produtoras no setor agrícola}

A estrutura de mercado do setor agrícola é de concorrência perfeita. Dessa forma, deve escolher a quantidade de insumos que maximiza o seu lucro:

$$
\max _{K_{t}^{a}, L_{t}^{a}} P_{t}^{a} C_{t}^{a}-R_{t}^{a} W_{t}^{a} L_{t}^{a}-R_{t} K_{t}^{a}
$$

sujeita à seguinte tecnologia motivada por Bragagnolo e Barros (2013) e Da-Rocha e Restuccia (2006):

$$
C_{t}^{a}=A_{t}\left(K_{t}^{a}\right)^{\alpha a}\left(A_{t}^{a} L_{t}^{a}\right)^{1-\alpha a}
$$

No setor agrícola existe dois tipos de tecnologia: uma do tipo Hicks-neutra, que possui a seguinte regra de movimento:

$$
\log A_{t}=\rho_{A} \log A_{t-1}+\varepsilon_{A, t}
$$

Em que $\rho_{A}$ é o componente autorregressivo dessa tecnologia e $\varepsilon_{A, t} \sim N\left(0, \sigma_{A}\right)$.

E outra tecnologia, que é aumentadora de trabalho:

$$
A_{t+1}^{a}=\left(A_{t}^{a}\right)^{1-\delta a} I A_{t}^{a}
$$

Em que $A^{\text {a }}$ é o estoque de conhecimento usado na agricultura $^{6}, \delta a$ é a taxa de depreciação desse conhecimento e $I A_{t}^{a}$ é o investimento em pesquisa neste setor que segue a regra de movimento:

$$
\log I A_{t}^{a}=\rho_{I A} \log I A_{t-1}^{a}+\varepsilon_{I A, t}
$$

Em que $\rho_{\text {IA }}$ é componente autorregressivo desse choque e $\varepsilon_{I A, t} \sim N\left(0, \sigma_{I A}\right)$.

As condições de primeira ordem para o problema anterior são:

$$
\begin{aligned}
& L_{t}^{a}=(1-\alpha a)\left(\frac{C_{t}^{a}}{\frac{R_{t}^{a} W_{t}^{a}}{P_{t}^{a}}}\right) \\
& K_{t}^{a}=\alpha a\left(\frac{C_{t}^{a}}{\frac{R_{t}}{P_{t}^{a}}}\right)
\end{aligned}
$$

As equações (19) e (20) representam as demandas por trabalho e capital no setor agrícola. Dado que este setor está em concorrência perfeita, o preço do bem agrícola será igual ao seu custo marginal:

$$
P_{t}^{a}=\left(\frac{1}{A_{t}\left(A_{t}^{a}\right)^{1-\alpha a}}\right)\left(\frac{R_{t}}{\alpha a}\right)^{\alpha a}\left(\frac{R_{t}^{a} W_{t}^{a}}{1-\alpha a}\right)^{1-\alpha a}
$$

E o custo do crédito deste setor $\left(R_{t}^{a}\right)$ é a combinação do crédito subsidiado e do crédito com remuneração de mercado:

$$
R_{t}^{a}=\theta^{\text {SUB }} R^{\text {SUB }}+\left(1-\theta^{\text {SUB }}\right) R_{t}^{N}
$$

\subsubsection{Firmas produtoras no setor não agrícola}

Diferente do setor agrícola, este setor possui certo grau de rigidez de preços; consequentemente, a estrutura de mercado é de concorrência monopolística. A forma de introduzir essa característica é dividir este setor nas produções de bens finais e de bens intermediários. Esse primeiro subsetor está em concorrência perfeita e apenas agrega os bens intermediários diferenciados.

\subsubsection{Firmas produtoras de bens finais no setor não agrícola}

Para produzir um bem agregado, esse tipo de firma compra uma grande quantidade de bens intermediários diferenciados para resolver o seguinte problema de maximização de lucro:

$$
\max _{Y_{j, t}^{n}} P_{t}^{n} Y_{t}^{n}-\int_{0}^{1} P_{j, t}^{n} Y_{j, t}^{n} d j
$$

6. Ideia baseada em Romer (1990). 
556 - Impacto das Variações no Crédito Rural e no Investimento em Pesquisa Agrícola na Produtividade da Agricultura Brasileira Contemporânea

sujeita a,

$$
Y_{t}^{n}=\left(\int_{0}^{1}\left(Y_{j, t}^{n}\right)^{\frac{\psi-1}{\psi}} d j\right)^{\frac{\psi}{\psi-1}}
$$

Em que $Y_{t}^{n}$ é o produto agregado não agrícola, $\psi$ é a elasticidade de substituição entre bens intermediários do setor não agrícola e $Y_{j, t}^{n}$ é o produto intermediário $j$ deste setor usado como insumo no problema da firma produtora de bens finais cujo preço é $P_{j, t}^{n}$.

A condição de primeira ordem para o problema anterior é:

$$
Y_{j, t}^{n}=Y_{t}^{n}\left(\frac{P_{t}^{n}}{P_{j, t}^{n}}\right)^{\psi}
$$

Substituindo a equação de demanda pelo bem $Y_{j, t}^{n}$ (equação (25)) na tecnologia dada pela equação (24), chega-se no nível de preços agregado.

$$
P_{t}^{n}=\left(\int_{0}^{1}\left(P_{j, t}^{n}\right)^{1-\psi} d j\right)^{\frac{1}{1-\psi}}
$$

\subsubsection{Firmas produtoras de bens intermediários no setor não agrícola}

O problema das firmas produtoras de bens intermediários é realizado em duas etapas. Na primeira, esta firma escolhe as quantidades de insumos que minimizam seu custo; em seguida, as firmas determinam o seu nível de preços. Dessa forma, nesse primeiro estágio, a firma deve resolver o seguinte problema:

$$
\min _{K_{t, j}^{n}, L_{t, j}^{n}} R_{t}^{N} W_{t}^{n} L_{t, j}^{n}+R_{t} K_{t, j}^{n}
$$

sujeita à seguinte tecnologia,

$$
Y_{t, j}^{n}=A_{t}^{n}\left(K_{t, j}^{n}\right)^{\alpha n}\left(L_{t, j}^{n}\right)^{1-\alpha n}
$$

Em que $\alpha$ n é participação do capital na produção de bens não agrícolas, e $A_{t}^{n}$ é o nível tecnológico deste setor, que possui a seguinte regra de movimento:

$$
\log A_{t}^{n}=\rho_{A n} \log A_{t-1}^{n}+\varepsilon_{A n, t}
$$

Em que $\rho_{A n}$ é componente autorregressivo desse choque $\varepsilon_{A n, t} \sim N\left(0, \sigma_{A n}\right)$.
As condições de primeira ordem para o problema anterior são:

$$
L_{t}^{n}=(1-\alpha n) C M_{t}^{n}\left(\frac{Y_{t}^{n}}{R_{t}^{N} W_{t}^{n}}\right)
$$

$$
K_{t}^{n}=\alpha n C M_{t}^{n}\left(\frac{Y_{t}^{n}}{R_{t}}\right)
$$

As equações (30) e (31) representam as demandas por trabalho e capital deste setor, respectivamente.

E o custo marginal do setor não agrícola é dado por:

$$
C M_{t}^{n}=\left(\frac{1}{A_{t}^{n}}\right)\left(\frac{R_{t}}{\alpha n}\right)^{\alpha n}\left(\frac{R_{t}^{N} W_{t}^{n}}{1-\alpha n}\right)^{1-\alpha n}
$$

\subsubsection{Precificação a la Calvo}

A firma produtora de bens intermediários deve decidir o preço do seu produto seguindo uma regra de Calvo (1983) em que há uma probabilidade $\theta$ de que as firmas mantêm o nível de preços do período anterior e a probabilidade $(1-\theta)$ de definir o preço do seu bem de forma ótima, $P_{t}^{n^{*}}$. Uma vez que o preço esteja definido em $t$, há a probabilidade $\theta$ de permanecer fixo em $t+1$, uma probabilidade $\theta^{2}$ de permanecer fixo em $t+2$, e assim por diante. Considerando isso, a firma capaz de ajustar preço deve resolver o seguinte problema:

$$
\max _{P_{t}^{n^{*}}} E_{t} \sum_{i=0}^{\infty}(\beta \theta)^{i}\left(P_{t}^{n^{*}}-C M_{t+i}^{n}\right) Y_{t+i}^{n}
$$

sujeita à equação (25).

Em que $\theta$ é o fator de rigidez no reajuste dos preços.

A condição de primeira ordem para o problema anterior é:

$$
P_{t}^{n^{*}}=\left(\frac{\psi}{\psi-1}\right) E_{t} \sum_{i=0}^{\infty}(\beta \theta)^{i} \mathrm{CM}_{t+i}^{n}
$$

Combinando a regra de precificação da equação (26) com a suposição de que todas as firmas em condições definem o preço da mesma forma, chega-se ao nível geral de preços para o setor não agrícola.

$$
P_{t}^{n}=\left[\theta\left(P_{t-1}^{n}\right)^{1-\psi}+(1-\theta)\left(P_{t}^{n^{*}}\right)^{1-\psi}\right]^{\frac{1}{1-\psi}}
$$




\subsection{Autoridade monetária}

Este agente possui duplo objetivo: nível do produto e estabilidade de preços. Para tanto, usa-se a seguinte regra de Taylor:

$$
\frac{R_{t}^{N}}{R_{s s}^{N}}=\left(\frac{R_{t-1}^{N}}{R_{s s}^{N}}\right)^{\gamma R}\left[\left(\frac{Y_{t}}{Y_{s s}}\right)^{\gamma \gamma}\left(\frac{\pi_{t}}{\pi_{s s}}\right)^{\gamma /}\right]^{1-\gamma R} S_{t}^{m}
$$

Em que $\gamma R$ é o parâmetro de suavização de alterações na taxa de juros, $\gamma Y$ e $\gamma \pi$ representam as sensibilidades da taxa de juros em relação ao produto e à taxa de inflação, respectivamente. $S_{t}^{m}$ é o choque de política monetária que segue a seguinte regra de movimento:

$$
\log S_{t}^{m}=\rho_{m} \log S_{t-1}^{m}+\varepsilon_{m, t}
$$

Em que $\rho_{m}$ é o parâmetro autorregressivo desse choque e $\varepsilon_{m, t} \sim N\left(0, \sigma_{m}\right)$.

\subsection{Condições de equilíbrio}

Para fechar o modelo são necessárias algumas condições de agregação:

$$
\begin{aligned}
& L_{t}=L_{t}^{a}+L_{t}^{n} \\
& K_{t}=K_{t}^{a}+K_{t}^{n} \\
& Y_{t}^{n}=C_{t}^{n}+I_{t} \\
& Y_{t}=Y_{t}^{n}+C_{t}^{a}
\end{aligned}
$$

\section{Estimação do modelo estrutural}

Nesta seção são apresentados os procedimentos de tratamento dos dados e de estimação do modelo estrutural.

\subsection{Tratamento dos dados}

Este modelo foi estimado usando dados anuais de 2005 até 2013. Foram usadas sete variáveis observáveis descritas no Quadro 1 e na Figura 2. Para preparar os dados para a estimação, as séries foram deflacionadas; em seguida, foi usado o software X12-ARIMA e aplicada a diferença dos logaritmos para retirar a sazonalidade e a tendência dos dados, respectivamente.

\subsection{Parâmetros calibrados, prior e posterior}

A calibragem dos parâmetros segue duas abordagens. Alguns parâmetros que não estão diretamente relacionados com o objeto principal deste trabalho foram calibrados, enquanto que os parâmetros relevantes para a análise da propagação dos choques são estimados usando uma metodologia bayesiana. Para a primeira abordagem, decidiu-se usar valores de parâmetros de outros artigos relevantes da literatura DSGE brasileira e estatísticas das variáveis observáveis. O Quadro 2 resume a calibragem desses parâmetros.

Dadas as distribuições priors dos parâmetros, o modelo foi estimado utilizando um processo de cadeia de Markov por meio do algoritmo Metropolis-Hastings

Quadro 1. Variáveis observáveis do modelo

\begin{tabular}{|c|l|c|}
\hline Variável & \multicolumn{1}{|c|}{ Série } & Fonte \\
\hline SUB & Operações de crédito ao setor privado - rural - R\$ (milhões) & Bacen \\
\hline $\mathrm{N}$ & $\begin{array}{l}\text { Soma de: } \\
\text { Operações de crédito ao setor privado - indústria; } \\
\text { Operações de crédito ao setor privado - comércio; e } \\
\text { Operações de crédito ao setor privado - outros serviços - R\$ (milhões). }\end{array}$ & Bacen \\
\hline Ca & PIB - agropecuária - ref. 2010 - R\$ (milhões) & IBGE/SCN \\
\hline I & Capital - formação bruta - ref. 2010 - R\$ (milhões) & IBGE/SCN \\
\hline IAa & Execução Orçamentária - Valor pago no ano & Embrapa \\
\hline Wa & Renda média de todos os trabalhos - áreas rurais - R\$ Outubro 2014 & IPEA \\
\hline RN & Taxa de juros - Over / Selic - (\% a.m.) & Bacen \\
\hline
\end{tabular}

Fonte: Elaboração própria. 
558 - Impacto das Variações no Crédito Rural e no Investimento em Pesquisa Agrícola na Produtividade da Agricultura Brasileira Contemporânea

Figura 2. Dados tratados
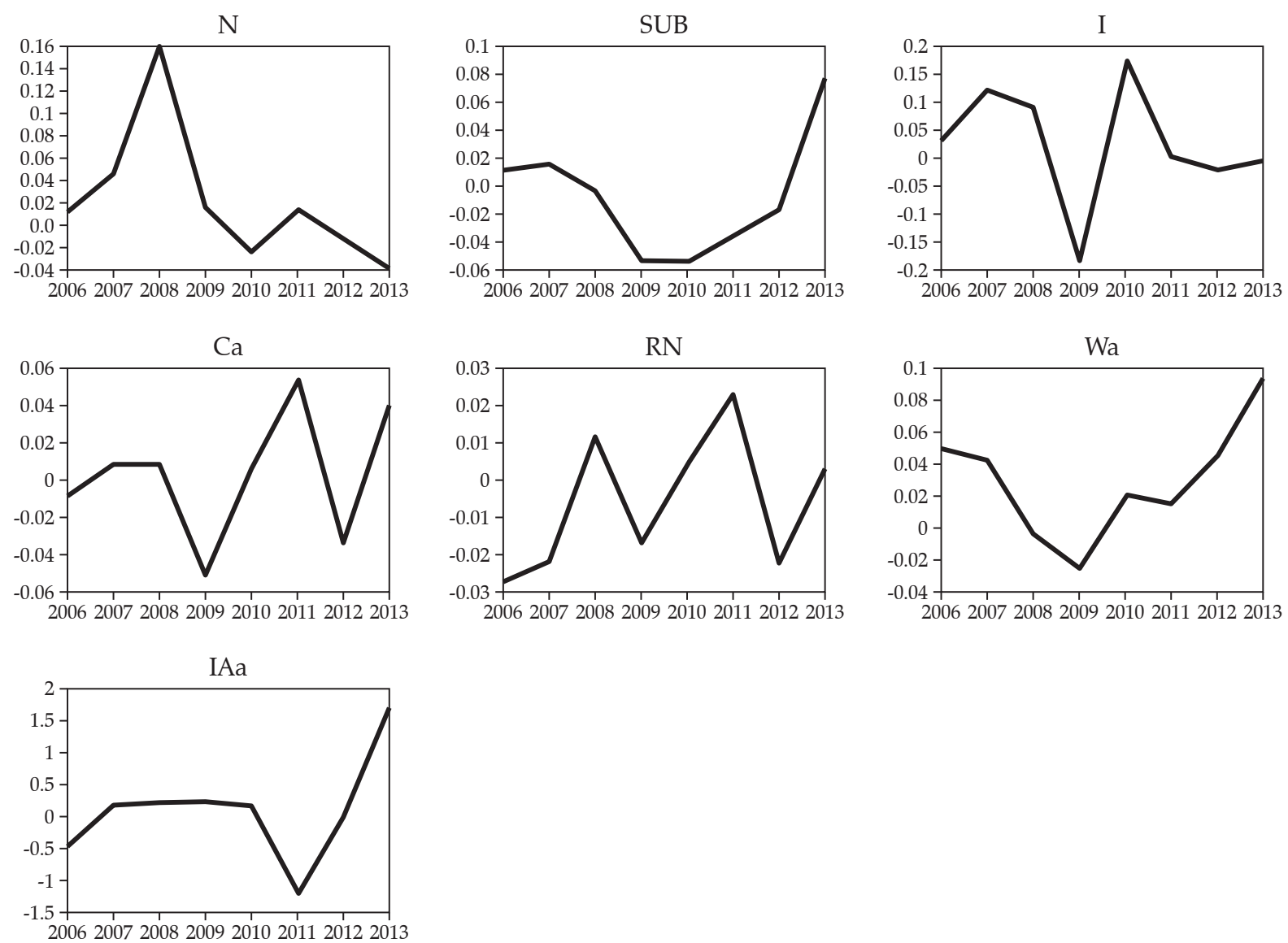

Fonte: Elaboração própria.

Quadro 2. Parâmetros calibrados

\begin{tabular}{|c|c|l|}
\hline Parâmetros & Valor & \multicolumn{1}{|c|}{ Fonte } \\
\hline$\beta$ & 0,875 & Referente à Selic de 14,25\% a.a. \\
\hline$\delta$ & 0,1 & Vereda e Cavalcanti, 2010 \\
\hline$\sigma$ & 2 & Vereda e Cavalcanti, 2010 \\
\hline$\varphi$ & 1,5 & Vereda e Cavalcanti, 2010 \\
\hline$\omega a$ & 0,08 & PIB - agropecuária/Consumo final - famílias \\
\hline$R^{\text {SUв }}$ & 1,0875 & $8,75 \%$ a.a. para operações de crédito rural a partir de 1/7/2015 \\
\hline
\end{tabular}

Fonte: Elaboração própria.

com 100.000 e 5 cadeias paralelas. Os resultados da estimação bayesiana estão apresentados na Tabela 1 e na Figura 3.

Os resultados da Figura 3 são relevantes pois apresentam os resultados da estimação deste trabalho. Para um bom resultado, é importante que as dis- tribuições priors e posteriors não sejam excessivamente diferentes uma da outra. O resultado da distribuição deve ser próxima de uma normal e a moda deve estar próxima da média. Dessa forma, analisando-se a Figura 3, percebe-se que os resultados obtidos foram satisfatórios. 
Tabela 1. Distribuição prior e posterior do modelo

\begin{tabular}{|c|c|c|c|}
\hline Parâmetros & Média prior & Média posterior & Intervalo confiança $\mathbf{9 0 \%}$ \\
\hline$\theta^{\text {SUв }}$ & 0,15 & 0,152 & $0,07 \quad 0,231$ \\
\hline$\psi$ & 10 & 9,56 & 513,86 \\
\hline$\theta n$ & 0,26 & 0,41 & $0,31 \quad 0,5$ \\
\hline$\alpha a$ & 0,75 & 0,75 & $0,7 \quad 0,791$ \\
\hline$\alpha n$ & 0,4 & 0,37 & $0,35 \quad 0,4$ \\
\hline$\gamma R$ & 0,75 & 0,66 & $0,6 \quad 0,72$ \\
\hline$\gamma Y$ & 0,3 & 0,25 & $0,1 \quad 0,42$ \\
\hline$\gamma \pi$ & 2,5 & 2,17 & 22,4 \\
\hline$\delta a$ & 0,11 & 0,07 & $0,01 \quad 0,17$ \\
\hline$A_{S S}^{a}$ & 1 & 1,02 & $0,72 \quad 1,34$ \\
\hline$\psi a$ & 12,5 & 12,68 & $10,76 \quad 15$ \\
\hline$\rho_{A}$ & 0,5 & 0,53 & $0,38 \quad 0,7$ \\
\hline$\rho_{A n}$ & 0,5 & 0,47 & $0,34 \quad 0,6$ \\
\hline$\rho_{I A a}$ & 0,5 & 0,52 & $0,35 \quad 0,72$ \\
\hline$\rho_{P}$ & 0,5 & 0,45 & $0,3 \quad 0,6$ \\
\hline$\rho_{L}$ & 0,5 & 0,66 & $0,54 \quad 0,78$ \\
\hline$\rho_{S U B}$ & 0,5 & 0,5 & $0,34 \quad 0,67$ \\
\hline$\rho_{m}$ & 0,5 & 0,28 & $0,16 \quad 0,4$ \\
\hline$\varepsilon_{A}$ & 1 & 0,46 & $0,24 \quad 0,68$ \\
\hline$\varepsilon_{A n}$ & 1 & 0,35 & $0,19 \quad 0,49$ \\
\hline$\varepsilon_{I A a}$ & 1 & 0,77 & $0,46 \quad 1,09$ \\
\hline$\varepsilon_{P}$ & 1 & 0,4 & $0,22 \quad 0,57$ \\
\hline$\varepsilon_{L}$ & 1 & 0,72 & $0,31 \quad 1,14$ \\
\hline$\varepsilon_{\text {SUв }}$ & 1 & 0,52 & $0,26 \quad 0,79$ \\
\hline$\varepsilon_{m}$ & 1 & 0,35 & $0,2 \quad 0,51$ \\
\hline
\end{tabular}

Fonte: Elaboração própria.

Figura 3. Priors e posteriors
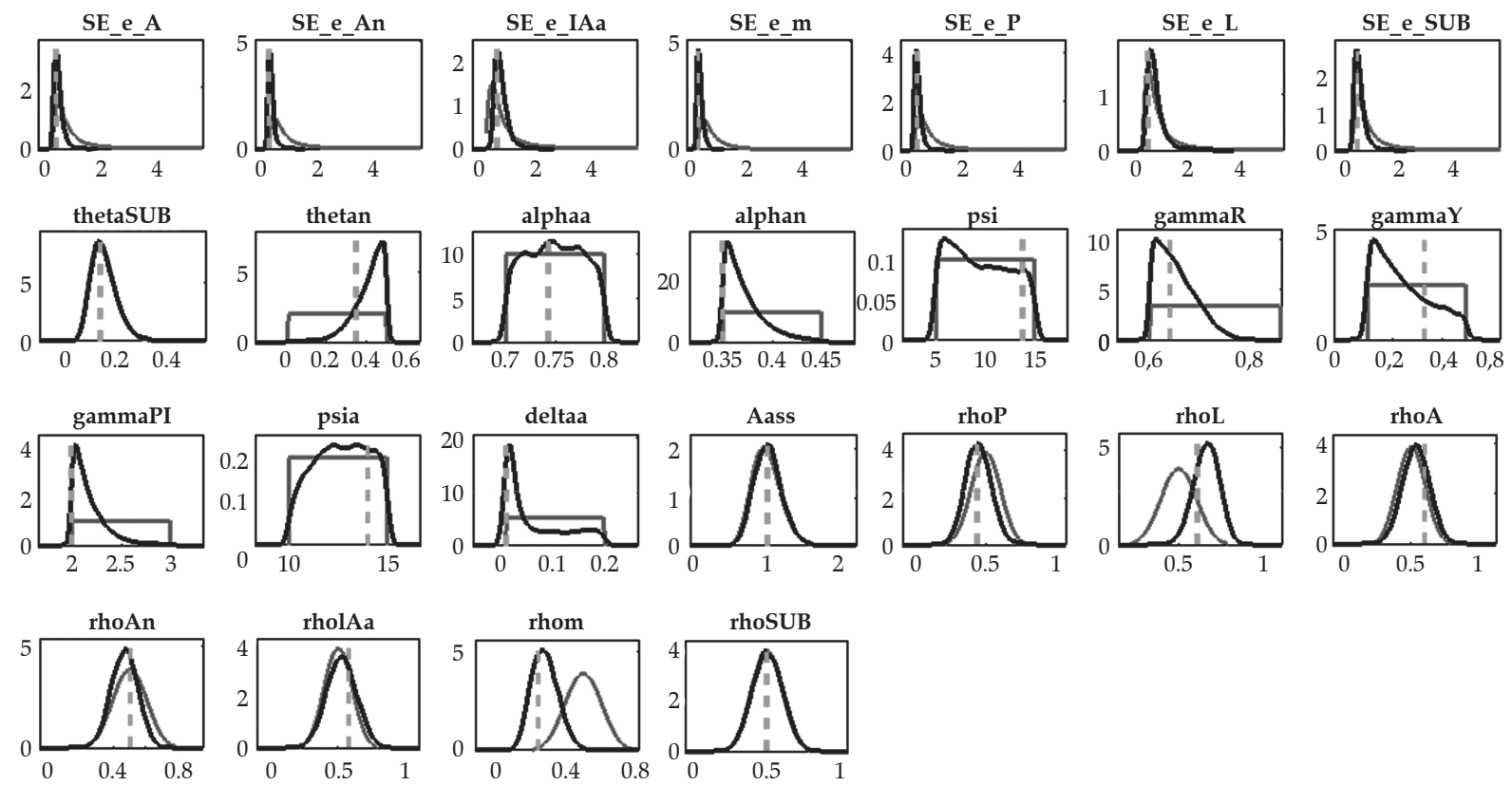

Fonte: Elaboração própria. 
560 - Impacto das Variações no Crédito Rural e no Investimento em Pesquisa Agrícola na Produtividade da Agricultura Brasileira Contemporânea

\section{Análise dos resultados}

A análise dos resultados deste trabalho envolve o estado das elasticidades da produção agrícola em relação à produtividade total dos fatores, à produtividade aumentadora do trabalho, aos insumos e ao crédito rural subsidiado. Também se verifica o comportamento da economia dados os choques no crédito subsidiado e no investimento em pesquisa agrícola. Esta análise é finalizada por meio de uma comparação das elasticidades da produtividade total dos fatores em relação ao crédito subsidiado e ao investimento em pesquisa agrícola deste trabalho com o trabalho de Gasques et al. (2012a).

\subsection{Análise das elasticidades em relação a produção do setor agrícola}

Esta subseção busca analisar a influência dos insumos, das produtividades e do crédito rural subsidiado no desempenho do setor agrícola no período de 2005 a 2013. Para tanto, inicia-se com a definição da elasticidade da produção do setor agrícola - variável usada nesta análise.

Definição 4.1 (Elasticidade da produção do setor agrícola): Registra a alteração proporcional no produto do setor agrícola em resposta a uma alteração proporcional em $X=\{A, A a, L a, K a, S U B\}$. Matematicamente,

$$
\text { elasCa_Xt }=\frac{\log \left(\frac{C a_{t}}{C a_{t-1}}\right)}{\log \left(\frac{X_{t}}{X_{t-1}}\right)}
$$

A Figura 4 apresenta o resultado das elasticidades da produção do setor agrícola para os insumos trabalho e capital, para a produtividade total dos fatores, para a produtividade aumentadora do trabalho e para o crédito rural subsidiado. Nota-se que a pro-

Figura 4. Elasticidades relacionadas a produção do setor agrícola
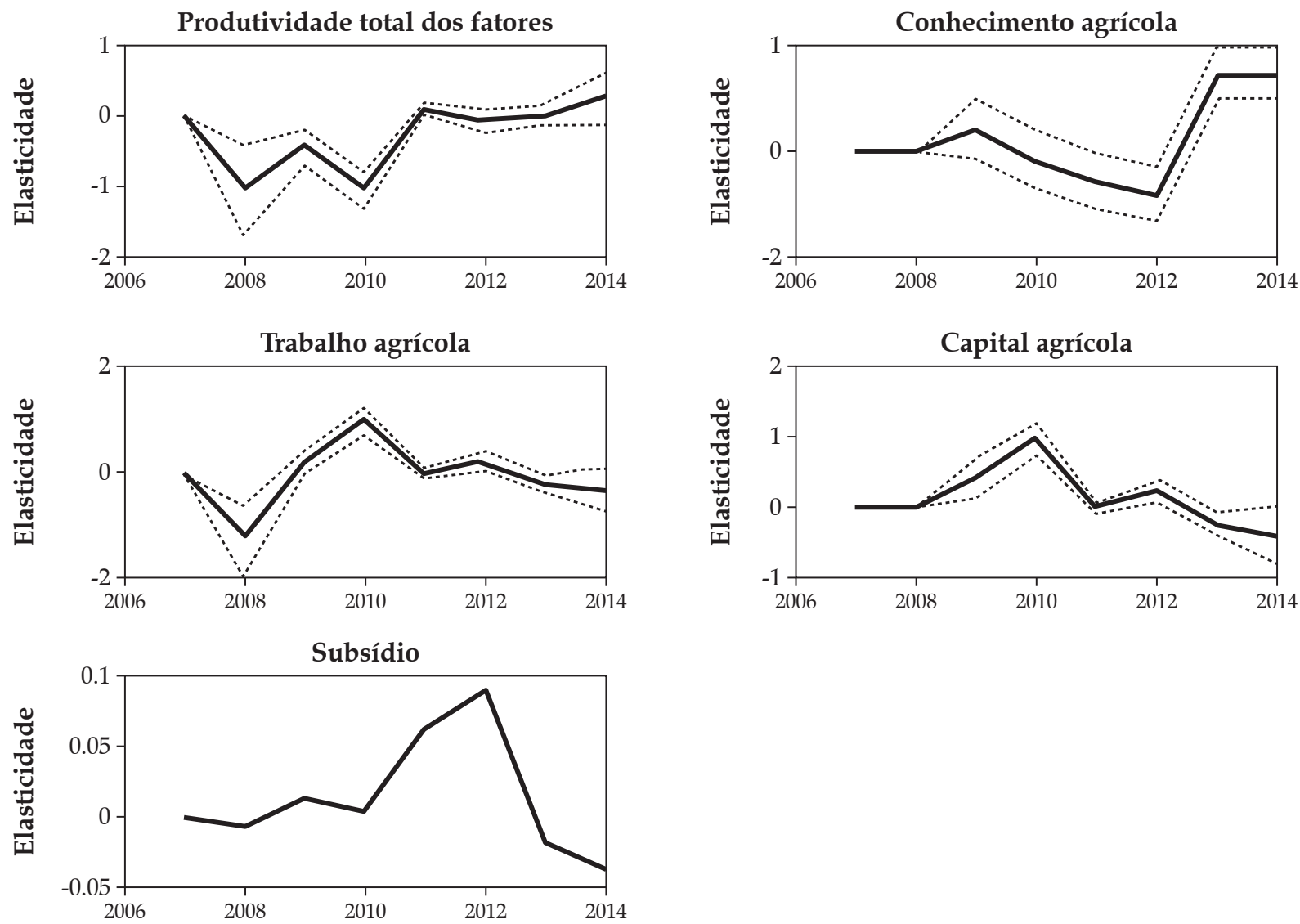

Fonte: Elaboração própria. 
dutividade total dos fatores tem contribuído negativamente para a produção agrícola, principalmente no período de 2006 até 2010, em que essa elasticidade foi negativa. A produtividade aumentadora do trabalho teve elasticidade negativa de 2009 até 2011, basicamente devido ao baixo investimento em pesquisa (Figura 2). A partir dessa data há uma inversão no comportamento do investimento em pesquisa e essa produtividade passa a ter contribuição significativa na produção agrícola.

Com exceção de 2007 - ano que os insumos trabalho e capital apresentaram elasticidades negativa e nula, respectivamente - o desempenho dos insumos na produção agrícola foi semelhante com valor positivo em 2009 e 2011, e negativo de 2012 até 2013. O crédito rural subsidiado teve a elasticidade praticamente nula até 2009, positiva entre 2010 e 2011 e negativa de 2012 até 2013.

Em resumo, a produtividade total dos fatores apresentou comportamento negativo até 2010, sinalizando que a queda de produtividade estava relacionada à falta de investimentos em pesquisa e à falta de crédito rural subsidiado.

\subsection{Choques no crédito rural subsidiado e na pesquisa agrícola}

Nesta subseção são apresentados os choques positivos no crédito rural subsidiado e no investimento em pesquisa agrícola.

A Figura 5, a seguir, apresenta funções impulso-resposta para um choque positivo no subsídio ao crédito destinado para o pagamento de salários no setor agrícola. Um crescimento no subsídio altera a alocação de capital da economia - o capital do setor agrícola aumenta, enquanto que o do setor não agrícola diminui. Isso ocorre pela necessidade de ajuste na restrição orçamentária das famílias, pois o crescimento do subsídio atua como um aumento "tributário" para as famílias. Esse agente precisa reajustar o seu orçamento destinando menos recursos para poupança - investimento e aquisição de ativos financeiros (empréstimos espontâneos para as firmas) diminuem. Essa falta de crédito espontâneo aumenta o custo financeiro das firmas, sugerindo um problema de má alocação dos recursos. Devido a esses fatos, o produto cai no primeiro momento; entretanto, em seguida apresenta um comportamento de retorno ao estado estacionário.
A Figura 6, a seguir, exibe o resultado de um choque positivo no investimento para pesquisa agrícola. O resultado é semelhante ao apresentado no aumento do subsídio, pois a renda disponível da família diminui e, dessa forma, diminui a poupança voluntária da economia. O diferencial é que o produto agregado neste choque retorna ao estado estacionário (e permanece neste nível) em dois períodos, enquanto que, no choque no aumento do subsídio, se recupera (superando o estado estacionário) também em dois períodos, retornando ao seu nível inicial no longo prazo. Em suma, pode-se pensar que o problema encontrado em ambos os choques é que, ao beneficiar um setor, o outro é prejudicado pelo canal da poupança voluntária. Esse problema é melhor trabalhado em Restuccia e Rogerson (2013) e em de Ferranti et al. (2005), cuja explicação seria que subsídios em setores específicos - como o agricultural - resultam na má alocação de recursos.

\section{Conclusões}

O objetivo deste trabalho é contribuir com uma análise do crédito rural subsidiado e do investimento em pesquisa agrícola, que estariam relacionados com o aumento da produtividade e o desempenho da agricultura brasileira. Para tanto, foi usado um modelo DSGE com economia fechada, sem governo e com dois setores, sendo que o setor agrícola incorpora a utilização de crédito rural subsidiado e pesquisa agrícola.

Os resultados apontam que o baixo investimento em pesquisa contribuiu com a baixa produtividade do setor agrícola até 2011, recuperando-se em 2012 e 2013. Por outro lado, o crédito rural subsidiado apresentou resultado nulo até 2009, resultado positivo em 2010 e 2011 e tornou-se negativo em 2012 e 2013.

As análises das funções impulso-resposta para os choques positivos no subsídio ao crédito rural e no investimento em pesquisa agrícola indicaram que esses choques alteram a alocação de capital da economia. Isso ocorre pela necessidade de ajuste na restrição orçamentária das famílias, pois os aumentos do subsídio e do investimento em pesquisa agrícola atuam como um "tributo" para as famílias no modelo. Essa falta de crédito espontâneo aumenta o custo financeiro das firmas, sugerindo um problema de má alocação dos recursos, fato discutido por Restuccia e Rogerson (2013) e por de Ferranti et al. (2005). Devido 
562 - Impacto das Variações no Crédito Rural e no Investimento em Pesquisa Agrícola na Produtividade da Agricultura Brasileira Contemporânea

Figura 5. Choque no crédito rural subsidiado

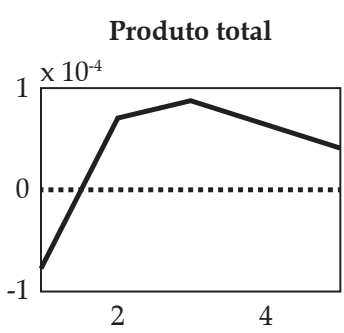

Produto agrícola

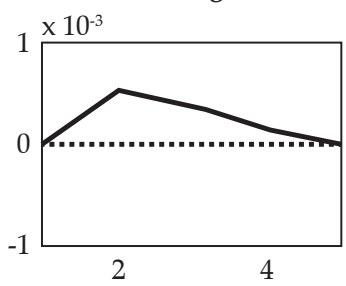

Capital total

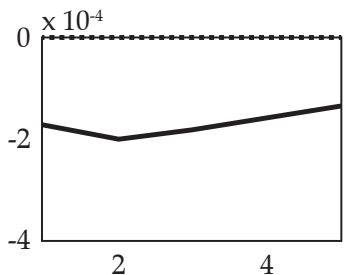

Remuneração das poupanças

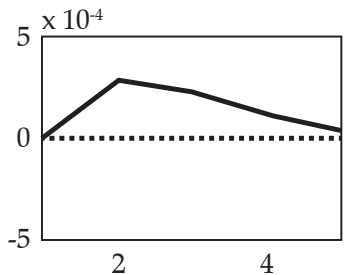

Salário agrícola

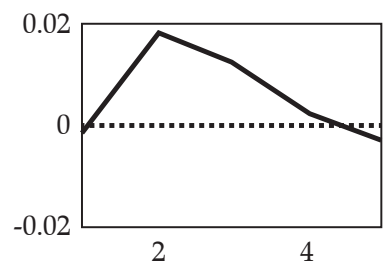

Produto não agrícola

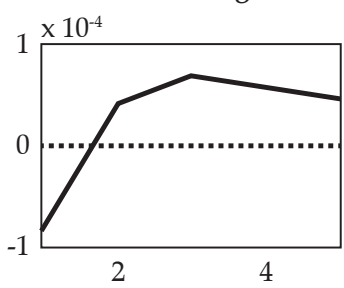

Consumo não agrícola

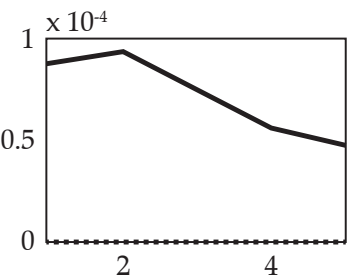

Trabalho agrícola

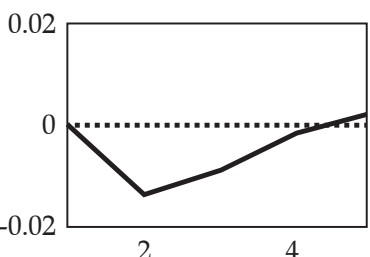

Taxa de inflação agrícola

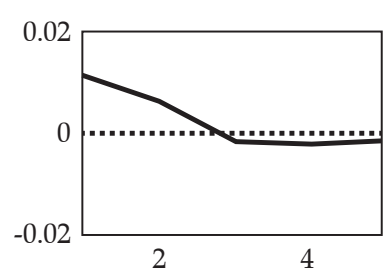

Salário não agrícola

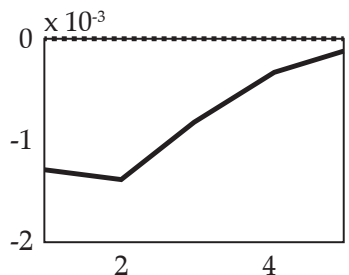

Consumo total

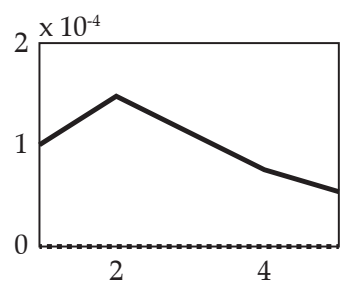

Capital agrícola

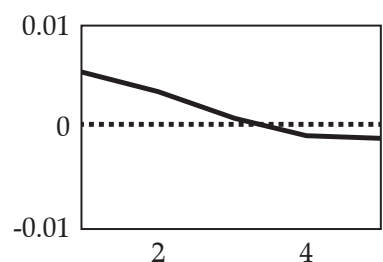

Trabalho não agrícola

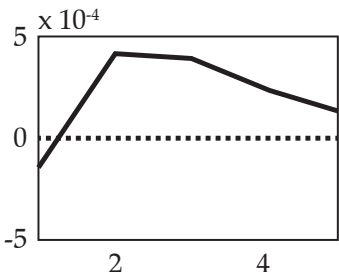

Taxa de inflação não agrícola $2 \times 10^{-3}$

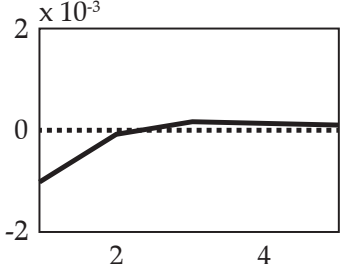

Poupança

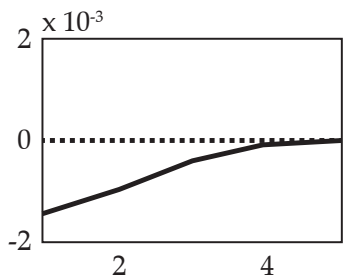

Investimento

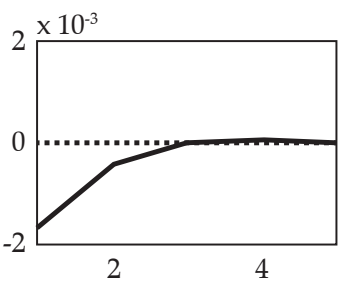

Capital não agrícola

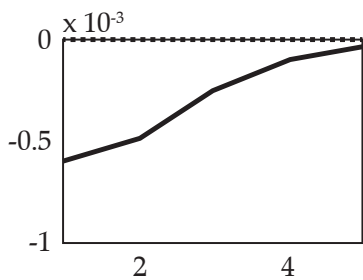

Trabalho total

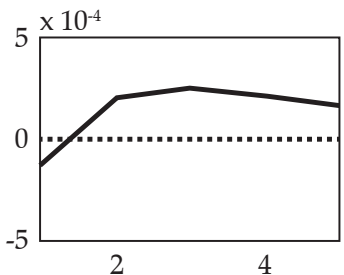

Taxa de inflação geral

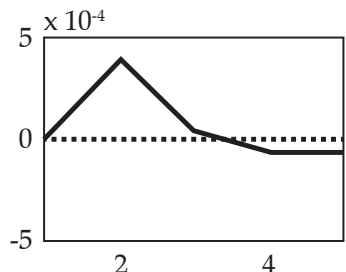

Subsídio

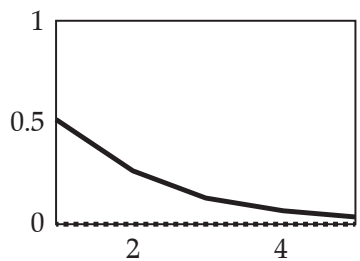

Fonte: Elaboração própria. 
Figura 6. Choque na pesquisa agrícola

Produto total

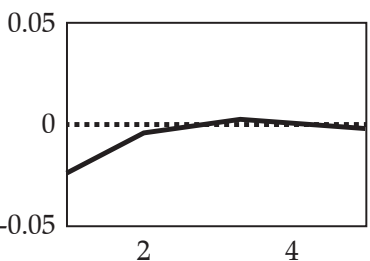

Produto agrícola

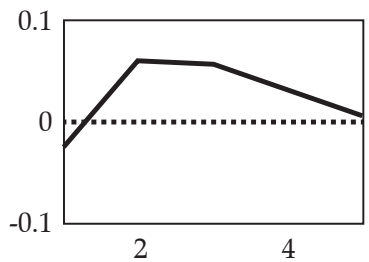

Capital total

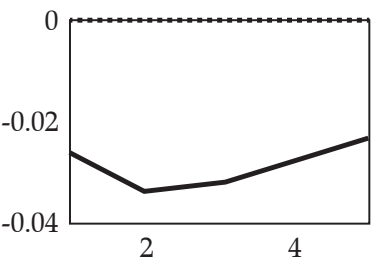

Remuneração das poupanças

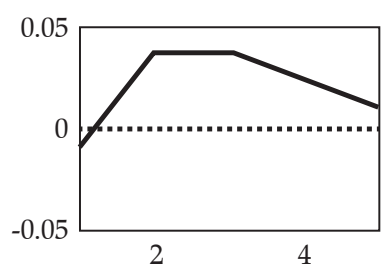

Salário agrícola

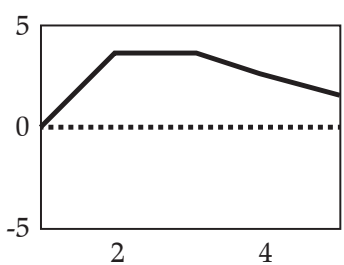

Investimento em

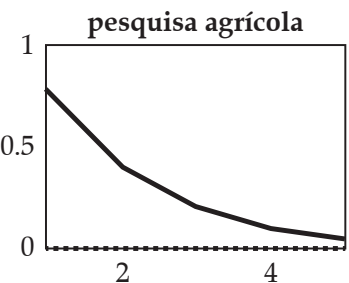

Produto não agrícola

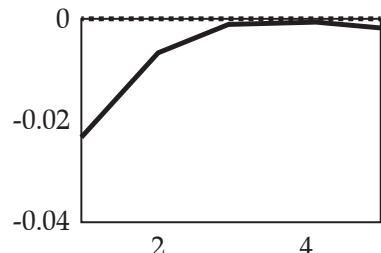

Consumo não agrícola

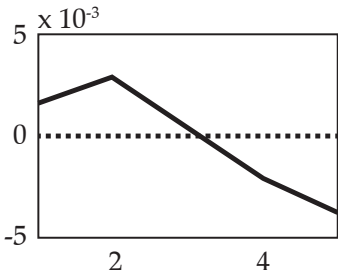

Trabalho agrícola

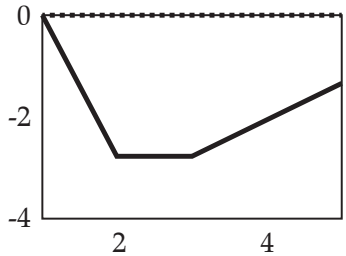

Taxa de inflação agrícola

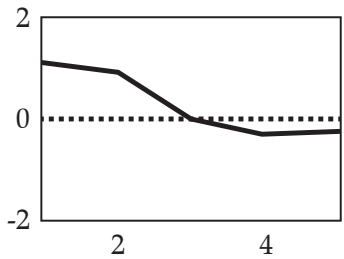

Salário não agrícola

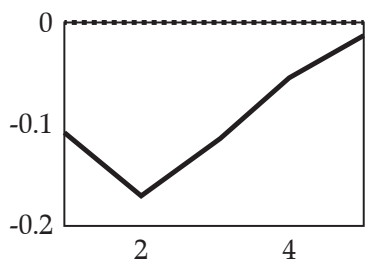

Conhecimento agrícola

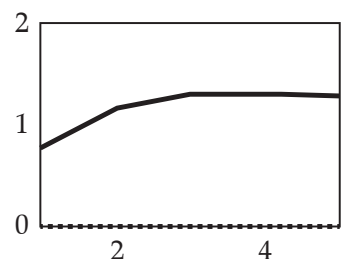

Consumo total

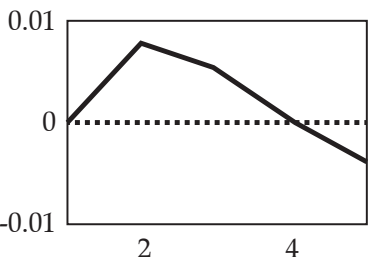

Capital agrícola

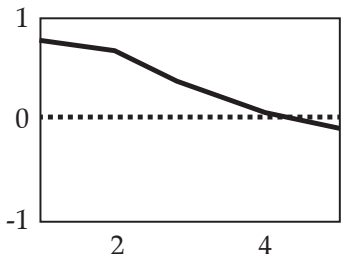

Trabalho não agrícola

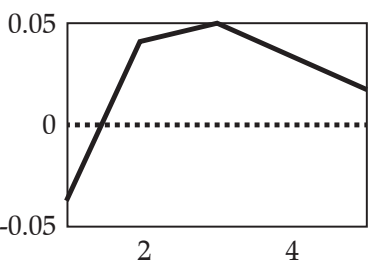

Taxa de inflação não agrícola

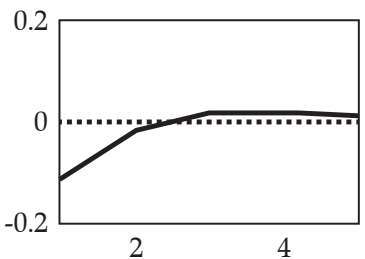

Poupança

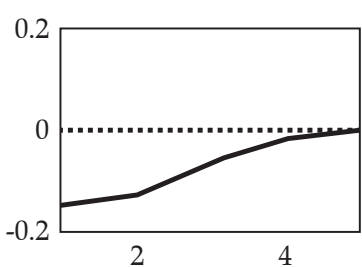

Investimento

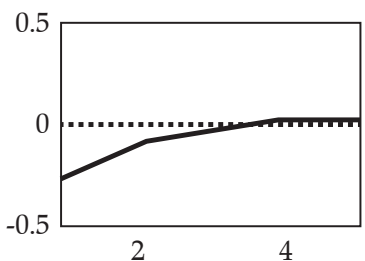

Capital não agrícola

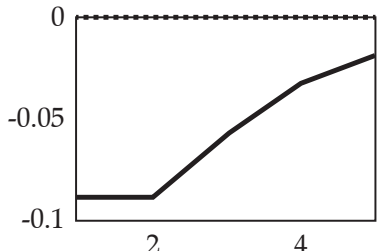

Trabalho total

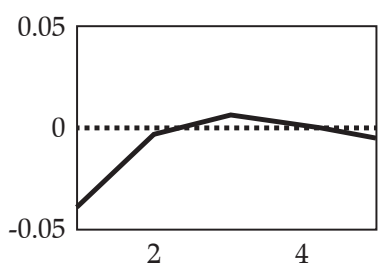

Taxa de inflação geral

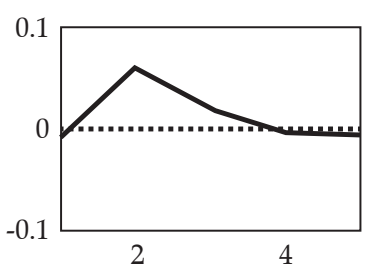

Subsídio

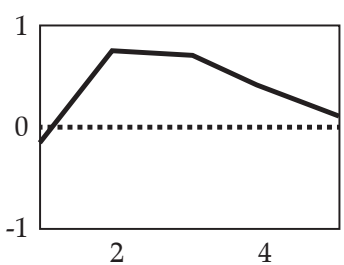

Fonte: Elaboração própria. 
564 - Impacto das Variações no Crédito Rural e no Investimento em Pesquisa Agrícola na Produtividade da Agricultura Brasileira Contemporânea

a esses eventos, o produto cai no primeiro momento; entretanto, em seguida apresenta uma recuperação, superando o nível de estado estacionário, no caso do subsídio ao crédito rural, e de retorno ao nível inicial, no caso do investimento em pesquisa agrícola.

Para pesquisas futuras, pode-se aprofundar na má alocação dos recursos, assumindo diferentes níveis para as produtividades dos setores.

\section{Referências}

BARROS, A. L. M. Capital, produtividade e crescimento da agricultura: o Brasil de 1970 a 1995. Tese (Doutorado em Economia Aplicada) - Escola Superior de Agricultura Luiz de Queiroz, (Esalq/USP), Piracicaba, 1999.

BONELLI, R. e FONTES, J. Desafios brasileiros no longo prazo. Textos para discussão, IBRE, 2013.

BragagnOlO, C. e BARROS, G. S. A. D. C. Ciclos econômicos na agricultura brasileira. Revista Brasileira de Economia, v. 67, n. 2, p. 151-175, 2013.

BRIGATTE, H. e TEIXEIRA, E. C. Determinantes de longo prazo do produto e da Produtividade Total dos Fatores da agropecuária brasileira no período 19742005. Revista de Economia e Sociologia Rural, v. 49, n. 4, p. 815-836, 2011.

CALVO, G. A. Staggered prices in a utility-maximizing framework. Journal of monetary Economics, v. 12, n. 3, p. 383-398, 1983.

CARLSTROM, C. T. e FUERST, T. S. Interest rate rules vs. money growth rules a welfare comparison in a cash-inadvance economy. Journal of Monetary Economics, v. 36, n. 2, p. 247-267, 1995.
COOLEY, T. F. e QUADRINI, V. A neoclassical model of the Phillips curve relation. Journal of Monetary Economics, v. 44, n. 2, p. 165-193, 1999.

DA-ROCHA, J. M. e RESTUCCIA, D. The role of agriculture in aggregate business cycles. Review of Economic Dynamics, v. 9, n. 3, p. 455-482, 2006.

DE FERRANTI, D. et al. Beyond the city: the rural contribution to development. Washington, DC: The World Bank, 2005.

FERREIRA, B. et al. Ocupações agrícolas e não-agrícolas: trajetória e rendimentos no meio rural brasileiro. In: NEGRI, J. A., NEGRI, F. e COELHO, D. Tecnologia, exportação e emprego. Brasília: IPEA, 2006, p. 445-488.

FREITAS, R. E. Produtividade agrícola no Brasil. In: DE NEGRI, F. e CAVALCANTE, L. R. Produtividade no Brasil: desempenho e determinantes. Brasília: ABDI, 2014, p. 373-401.

FUERST, T. S. Liquidity, loanable funds, and real activity. Journal of monetary economics, v. 29, n. 1, p. 3-24, 1992.

GASQUES, J. G. et al. Produtividade da agricultura brasileira e os efeitos de algumas políticas. Revista de Política Agrícola, v. 21, n. 3, p. 83-92, 2012a.

. et al. Total factor productivity in Brazilian agriculture. Productivity Growth in Agriculture: An International Perspective. CAB International, Oxfordshire, UK, 2012b, p. 145-162.

HERRMANN, L. F. Changes in agricultural production in Brazil, 1947-65 (No. 79). Economic Research Service, US Department of Agriculture, 1972.

ROMER, P. M. Endogenous technological change. Journal of Political Economy, v. 98, (5 pt 2), 1990.

Todo o conteúdo deste periódico, exceto onde estiver identificado, está licenciado sob uma Licença Creative Commons (cc by 4.0). 